\title{
SURO-LC: a space-based ultra-long wavelength radio observatory
}

\author{
Willem A. Baan \\ Netherlands Institute for Radio Astronomy, Dwingeloo, the Netherlands \\ E-mail: baaneastron.nI
}

\begin{abstract}
The space-based ultra-long wavelength radio observatory SURO-LC is a mission concept for a multi-satellite observatory constellation with $30 \mathrm{~km}$ in diameter positioned at the second SunEarth Lagrangian Point. This interferometric radio observatory operates below $60 \mathrm{MHz}$ and particularly in the range of $0.5-30 \mathrm{MHz}$ where the Earth's ionosphere absorbs and modifies all external emissions. SURO-LC is a Small-Class mission consisting of a mini-satellite Mothership and eight nano-satellite Daughters equipped with an omni-directional antenna system capable of observing all the sky all the time. The Mothership will serve as a control and processing center that communicates with the earth stations. The constellation will perform a loose formation flying operation where the positions of the Daughters are not fixed but only need to be known. The science case for SURO-LC covers a wide range of scientific disciplines including cosmology, extragalactic astronomy, Galactic astronomy, heliophysics and space weather, and planetary radio physics.
\end{abstract}

From Antikythera to the Square Kilometre Array: Lessons from the Ancients,

12-15 June 2012

Kerastari, Greece 


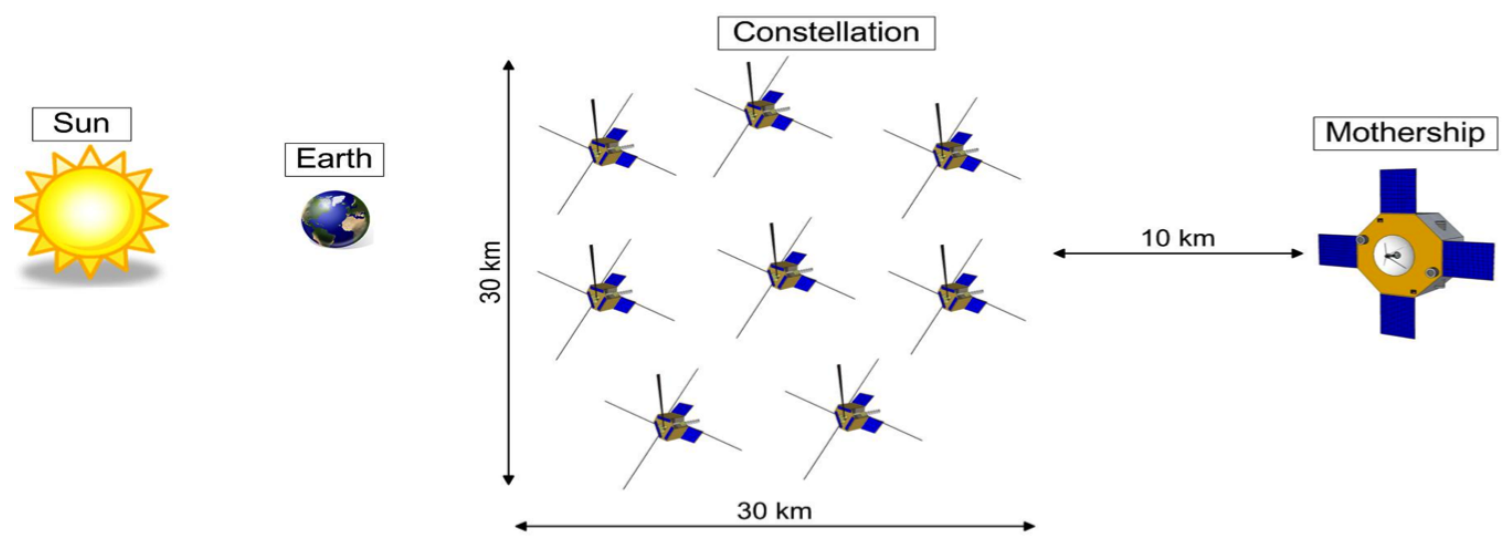

Figure 1: Constellation plan of SURO-LC at the Sun-Earth $2^{\text {nd }}$ Lagrangian point at $1.5 \mathrm{M} \mathrm{km}$ away from Earth. SURO-LC has eight (or more) nano-satellite Daughters equipped with three orthogonal dipole lowfrequency receiving antennas and a mini-satellite Mothership. The Daughters observe omni-directionally at frequencies below $60 \mathrm{MHz}$. The Mothership processes the observing data and connects with Earth.

\section{Motivation for a research project}

In scientific research, much of the learning process is based on acquiring the building blocks that can be used to reach a higher objective. Learning processes and developing scientific and technical insights invoke the use of these available building blocks to facilitate the next step in development that is obvious or clear or risky or even unbelievable. Progress results from being able to take this small next step or that giant leap.

The Antikythera Mechanism is an example of taking the next step in visualizing and interpreting the observational knowledge of the heavens. The Mechanism combines the building blocks from the diverse fields of astronomy, mathematics, metalworking and mechanical techniques, in order to make a step forward. The rewards for such activities may be material, financial, purely scientific, or, at a minimum, satisfying one's curiosity.

The SURO-LC project is a modern example of putting many building blocks together to reach a higher objective. SURO-LC is a Space-based Ultra-long wavelength Radio Observatory, at Low Cost for a space mission. While this concept has been proposed several times, dating back as early as 1977 , it did not get accepted by the space agencies because the existing technology was not considered to be sufficient for such a complex mission. It is only recently that the proper tools have become available to make this a reality. A detailed description of the SURO-LC proposal can be found on the internet [4].

In the following sections, the SURO-LC research objectives as well as the technical components of such a mission will be considered.

\section{The Science Objectives}

The motivation or the 'science case' of a space-based SURO-LC observatory is to perform radio observations at frequencies below $60 \mathrm{MHz}$ and particularly in the $0.5-30 \mathrm{MHz}$ range, where 


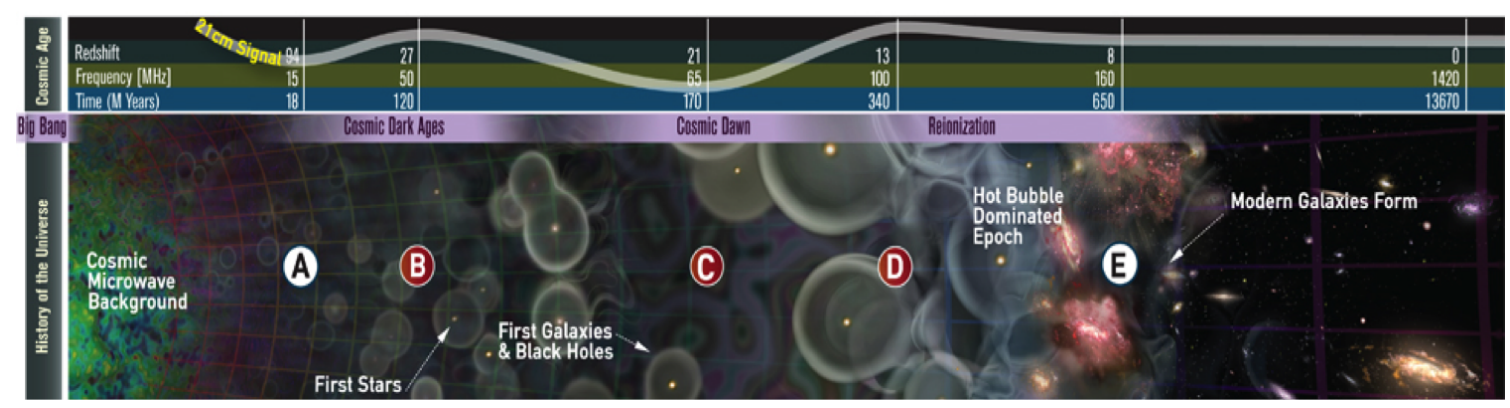

Figure 2: History of the Early Universe versus redshift, the redshifted $21 \mathrm{~cm}$ frequency, and time. The distinct stages of ionization have been identified in the text $[1,3]$.

the Earth's ionosphere strongly absorbs and modifies the signals for terrestrial observatories. This frequency range has not been explored extensively by the science community and a radio observatory in this range would strongly complement and supplement the capabilities of terrestrial radio instruments. The constellation plan for this observatory is depicted in Figure 1. The scientific studies by SURO-LC [4] at low frequencies cover a wide range of scales and physical phenomena.

All Sky Surveys - A spatial array of a $30 \mathrm{~km}$ diameter will produce omni-directional imaging with 3 arcmin resolution and $30 \mathrm{mJy}$ sensitivity at $10 \mathrm{MHz}$, and 1 arcmin and $20 \mathrm{mJy}$ sensitivity at $30 \mathrm{MHz}$. All-sky surveys will detect 2 million sources during a one year data collecting period. Surveys followed by studies of individual sources will allow the study of (1) radio source populations and their long-term evolution, and (2) the role of active galactic nuclei (AGN) and the feedback they give to the host galaxies. Individual sources of particular interest to be studied among the survey populations are relic radio sources, sources with a restart of nuclear activity (expected to be numerous at redshift $\mathrm{z}=20$ ), and the holes created in the intra-cluster medium by radio sources activity (expected to be some $10^{5}$ sources).

Cosmology: the Dark Ages and the Epoch of Reionization - The well known $21 \mathrm{~cm}$ transition of neutral hydrogen provides an accurate tracker of the ionization history during the Dark Ages of the universe. The (weak) emission or absorption strength of the $21 \mathrm{~cm} \mathrm{HI} \mathrm{line} \mathrm{varies} \mathrm{as} \mathrm{a} \mathrm{function}$ of redshift and this variation determines the timing and duration of the distinct evolutionary stages of the early universe (Fig. 2).

During Stage A of the Cosmic Dark Ages until redshift $\mathrm{z}=40$, the hydrogen in the universe continues to absorb the cosmic background (CMB) radiation field.

During Stage $B$ of the Cosmic Dark Ages, the star formation is ignited and at $\mathrm{z}=30$ the CMB photons and UV photons from the first generations of stars are absorbed by cold HI.

During Stage $C$ of the Cosmic Dawn, the first black holes formed at $\mathrm{z}=20$ and the first Galaxies and accreting black holes create HI bubbles in the intergalactic medium (IGM).

During Stage $D$ during Reionisation around $\mathrm{z}=13$, the stars and galaxies make hot ionized bubbles in the IGM that emit HI 21-cm emission.

Finally, at Stage E there is complete ionization of the IGM by stars and galaxies after which HI 21-cm signature disappears.

SURO-LC observations will cover the redshift range from 20 to over 1000 and will complete 


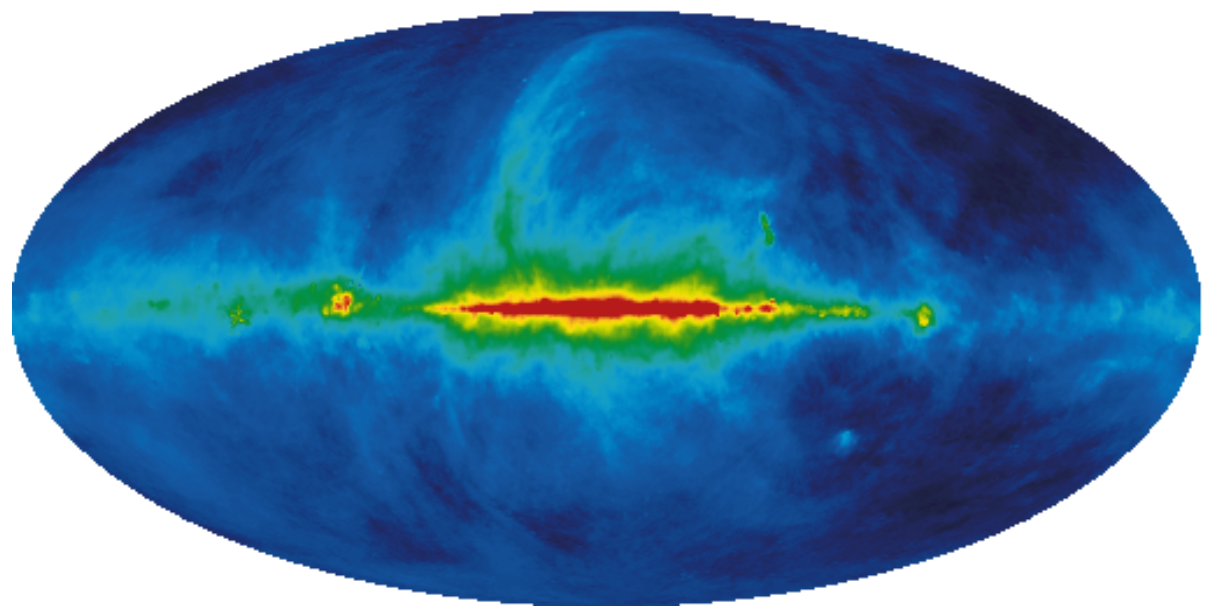

Figure 3: Low frequency radiation in the Galaxy at $408 \mathrm{MHz}$. Clearly visible in this map are the Galactic plane with specific activity areas as well as the Magellanic Stream reaching out from the plane towards the Large and Small Magellanic Cloud galaxies within our Local Group. This map has been made from a mosaic of spot measurements by the Effelsberg telescope (Germany) and the Parkes telescope (Australia) [2]. SURO-LC will be able to observe all the sky all the time.

the story of the Dark Ages after ground-based experiments measure its signature at lower redshifts.

HelioPhysics and Solar Weather - Solar studies at lower frequencies are of great import in understanding fundamental solar processes, but they are limited by the Earth's ionosphere. SUROLC with a baseline of $30 \mathrm{~km}$ provides unobstructed radio imaging capability of Type II and III bursts with 3 arc minutes resolution at $10 \mathrm{MHz}$, which is equivalent to that of terrestrial instruments at 70 $\mathrm{MHz}$ and higher. In addition, SURO-LC will be able to image and follow Coronal Mass Ejections (CMEs) to much larger distances from the Sun and beyond the Earth's orbit. Solar observing capability of SURO-LC augments and completes terrestrial research endeavors. SURO-LC would further a range of multi-wavelength studies of the Sun and the Solar Weather.

Planetary Studies - The radio planets in our solar system display strong magnetospheric emissions that are triggered by solar activity and the passing of CME outflows. Jupiter, Saturn and Uranus display their strongest emissions with very complex spectral structures in the $0.1-20 \mathrm{MHz}$ range of SURO-LC. Consequently, SURO-LC will be the instrument of preference and will constitute a platform not only for the detailed studies of these known planetary sources but also for Jupiter-like exoplanets in other planetary systems.

Galactic Studies - At low frequencies the structure and properties of the Galactic interstellar medium (ISM) are only partially known and largely unexplored. The Galactic emissions create a complex radio structure in and above the plane of the Galaxy (Fig. 3) containing the signatures of discrete sources and the diffuse ISM comprising the Galaxy. This local emission forms a background to be subtracted for studies of emissions originating outside the Galaxy. The local clumpy warm ionized medium (WIM) inside the ISM merits much study still, since the earlier and only low-frequency studies with the RAE-2 (1975) and IMP-6 (2002) satellites.

The 3D origin of cosmic rays and its connection to nearby HII regions and supernova remnants lie buried in the Galactic emissions. Discrete sources to be studied are some strong pulsars, 
whose low frequency emission properties are not at all understood, and transient sources, considered numerous, of still unexplored origins. Radio recombination lines originate in the ISM and form a foreground component to be subtracted from the low frequency data obtained in order to study extragalactic sources and to detect the Epoch of Reionization.

Observing Priorities - The objectives of SURO-LC derived from these science objectives are the following: (1) to optimize existing antenna performance using three orthogonal dipoles to achieve omni-directional observing and imaging capability, (2) to acquire data at the highest possible data rate, (3) to employ multiple satellite stations to utilize tried and true terrestrial (and space) interferometry techniques, (4) to use newly developed formation flying control and ranging techniques, (5) to reduce the radio background component and the interference (RFI) environment by positioning the system away from the Earth, (6) to select a stable gravitational environment (with small gravity gradients) at the Sun-Earth $2^{\text {nd }}$ Lagrangian point, and (7) to observe all the sky all the time.

\section{Putting the Components Together}

Technical and operational techniques obtained from diverse fields are required to put a SUROLC (low cost) space mission together: orbital mechanics, macro and micro-propulsion, communication and telemetry techniques, computing, imaging techniques, light-weighting construction, attitude control and formation flying control systems, antenna techniques, and many more.

The Constellation - The concept of the SURO-LC constellation resulting from the large-scale requirements is depicted in Figure 1. Located at the Sun-Earth $2^{\text {nd }}$ Lagrangian point, SURO-LC will consist of eight (at least) Daughter satellites and a Mothership. While the whole constellation drifts away (very) slowly from the Sun and Earth in a so-called Lissajous (spiral) orbit, the Mothership holds a 'fixed' position at $10 \mathrm{~km}$ from the Daughters. In addition, the Daughters will slowly drift back and forth through the spherical constellation with a diameter of about $30 \mathrm{~km}$, thus continuously changing the station configuration, which is good for the system's imaging performance. The number of Daughters has been limited to eight by launch weight considerations. More Daughters would give more daughter-daughter baselines and even better imaging.

The Lissajous orbit at L2 is selected for two properties: a very quiet radio environment and low relative inter-satellite drift rates. The stable trajectories requiring minimal corrective propulsion to counter the drift away from the saddle point provide a useable condition for SURO-LC in order to operate for three or more years.

Each of the Daughters serves as an observing station and is equipped with three dipole antennas with known performance characteristics. These three dipoles allow omni-directional observing and imaging of the entire sky using radio interferometry techniques that are used daily in terrestrial observatories around the world and in global positioning systems. The Mothership carries the correlator for processing the collected data from the Daughters and serves as a relay station for the correlated data to Earth.

Loose Formation Flying - In order for radio interferometry techniques to be effective, the relative positions of each of the Daughter satellites must be known to a fraction of the observing wavelength, or about $1 \mathrm{~m}$ for frequencies around $30 \mathrm{MHz}$. Modern metrology and sensing 


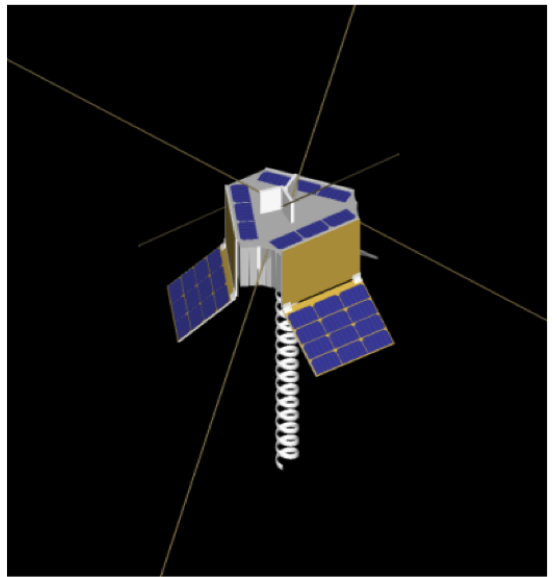

Figure 4: The simplified drawing of the envisioned Daughter satellite based on the SST SNAP nano satellite design. All unneccessary functionality will be stripped from the platform in order to reduce weight. The triangular design promotes stabilization in the solar wind (from above). The three orthogonal dipole antennas are mounted on the sides of the spacecraft. The high-gain antenna serves for data transfer to the Mothership.

techniques and a closed-form computing algorithm, together with the attitude and orbital control system, enable the determination of distances between all satellites to the required accuracy.

The formation flying requirements used for SURO-LC are relaxed in the sense that the Daughters do not need to be in prescribed positions (at high accuracy) as required for other formation flying proposals. Rather the Daughters are free to drift throughout the constellation as long as it is known where they are. Some control of the trajectories is required for collision avoidance and for nudging the Daughters back when they reach the outer edge of the constellation.

Small Satellite Technology - Small satellite technology will be used for the Daughters and the Mothership. The Daughters will be nano-satellites with a mass of only about $10 \mathrm{~kg}$, while the Mothership will be a mini-satellite of less than $500 \mathrm{~kg}$. Minimizing the mass of the cargo while maintaining system capabilities is a challenge for all space missions because of launch costs. The SURO-LC satellites will be stripped versions of existing satellite platforms such as the SNAP nano-satellite (SSTL), eliminating all non-essential components, and using lightweight (minimized) component options (concept drawing in Fig. 4). The Mothership will be a stripped version of the LISA PathFinder spacecraft.

The Daughter spacecrafts are kept pointing at the Sun mainly by passive attitude control using the radiation pressure on the slanted solar panels. Back-up miniature reaction wheels using recent technology solutions will provide the initial orientation control prior to the deployment of the solar panels, and allow active attitude control during propulsion manoeuvres.

Communication - Besides the metrology and ranging systems and the telemetry system that connects all satellites with each other, the transfer of the observational data from Daughters to Mothership requires the highest possible data rate. The rapidly developing android technology used in mobile communication systems $(2.4 \mathrm{GHz})$ provides the required data transfer for the intersatellite data links (ISLs) between the satellites (rate $>60 \mathrm{Mbps}$ at $0.7-1 \mathrm{GHz}$ ). The Mothership supports the communication up-down link with the system control center on Earth and maintains the data downlink for the condensed observing data at standard allocated frequencies.

On-board Signal Processing - The newest hardened high-power processing chips will be used in the Daughters and Mothership. Processing at each of the Daughters may be used for data conditioning and RFI mitigation algorithms in order to reduce the effects of terrestrial radio interference, which also reduces the required data rate for transfer to the Mothership. Correlation 

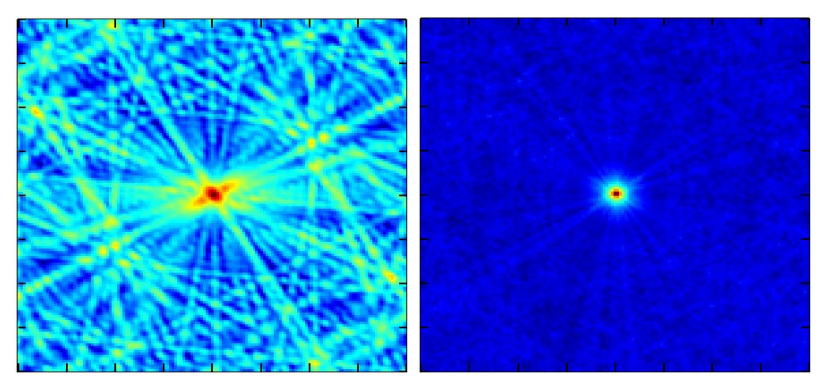

Figure 5: The point spread function (PSF) for a random 8 station SURO-LC with a $40 \mathrm{~km}$ baseline. a) A single snapshot PSF after averaging 10 frequency bins over $10 \mathrm{MHz}$ (bandwidth synthesis). The spoked sidelobes will scale with frequency. b) The PSF after 52 snapshots with random configurations (one snapshot per week for a year). The side-lobe dynamic range for these images is respectively 8 and 20 . Field of view is $2 \times 2$ degrees

processing in the Mothership will consider the observing data from each of the pairs (28 pairs for 8 Daughters) and produce a matrix of all correlation products. This reduces the data by a further factor of 120. On-board data processing is essential in order to reduce the downlink data rate of the system and to optimize the science return of SURO-LC.

Antenna Technology - The three orthogonal dipole antennas for all-sky imaging are mounted on the side surfaces of the Daughter satellites. Similar dipole systems are currently being used in terrestrial instruments operating in the $50-100 \mathrm{MHz}$ range (only for one hemisphere), such as LOFAR (Netherlands and Europe), and the Murchison Widefield Array (MWA, Western Australia). Since all radiation from all directions in the sky reaches all six antennas and is being recorded, the antennas' signals can later be phased-up to form a particular beam for observing a certain direction in the sky. This imaging process happens after the data has been received, collected, and integrated at the ground station.

Interferometric Imaging - The observational data collected during the mission will be stored in order to be used for interferometric imaging of the radio sky. Each of the correlation matrices produced during the mission represents a single snapshot observation using all Daughters in a particular configuration. The instantaneous imaging capability of the instrument with 8 Daughters is still quite limited and is described with a point spread function (PSF). However, the point spread function of the system improves when adding together many snapshots from the slowly moving constellation (and possibly more satellites), which will allow accurate imaging as shown in Figure 5. The imaging of the sky is done piece-by-piece by phasing up the available data to a particular position in the sky and making a small map of that area. These small maps will later be put together to form a mosaic of the sky.

The imaging objective of SURO-LC is to produce an image data product of the whole sky to the highest resolution after one year, two years, and three years of data has been collected. This procedure also incorporates the imaging of individual sources of interest. These imaging procedures and the cleaning of images from instrumental or other artifacts have become an art form for the users of terrestrial radio-imaging instruments. All this expertise will be directly applied to the SURO-LC data products.

Launch Procedures - SURO-LC will be launched with a VEGA launch vehicle that will place the Mothership and its cargo of 8 Daughter satellites (dry mass $600 \mathrm{~kg}$ and wet launch mass 1880 $\mathrm{kg}$ ), into a 200x1600 km orbit. In a similar manner to the LISA PF, the spacecraft uses its chemical main engine to perform the manoeuvres required to reach a Lissajous orbit at the second Sun-Earth 
Lagrange point (L2).

To prevent excessive relative initial drift rates, the Mothership places each Daughter sequentially in a parallel orbit. After the Mothership with all Daughters attached has reached a stable orbit, the mechanical separation device for launch and transit is jettisoned. The Daughters remain attached to the Mothership until the disturbance rates are minimised using the Mothership's micropropulsion system. Then the attachment mechanism releases the Daughters and the Mothership and Daughters will gently manoeuvre away from each other. The precision manoeuvring for the whole observatory system in 6 degrees of freedom is thus contained in the Mothership and does not have to be replicated in each Daughter. This type of disturbance-free separation system has been derived from nano-satellite and Cubesat delivery systems.

\section{Epilogue}

The SURO-LC space observatory represents a new way of putting together existing scientific knowledge and technical know-how. Because the lowest-frequency spectral region still represents an almost virgin territory for radio science, a SURO-LC radio observatory will have a great opportunity to make fundamental discoveries in a wide range of radio science research.

The SURO-LC project will certainly move current boundaries of scientific understanding by making new discoveries, unlike the objective of the Antikythera Mechanism, which served to visualize the already discovered 'operation' of the heavens.

The larger scale of the SURO-LC project requires many contributors. Besides the core team members from two research institutes (NL and SE), two consulting companies (UK), and one satellite technology company (UK), there were the Principal Investigator, ten national Co-Investigators, and a worldwide team of 90 scientists representing all research areas from 17 countries [4].

The SURO-LC concept has been submitted as a Small-Class mission proposal to ESA, the European Space Agency. Irrespective of the outcome, SURO-LC is a project whose hour has come and the international science community will certainly accept this challenge.

\section{Acknowledgements}

The authors thanks the SURO-LC Management Team, the Support Engineering Team, CoInvestigators, and the supporting scientist for continued enthusiastic support for putting the proposal together. Thank you especially: David Robinson (Psi-tran Ltd., UK), Richard Blott (SEP, Space Enterprise Partnerships, UK), Albert-Jan Boonstra (ASTRON, Netherlands Institute for Radio Astronomy, NL), Jan Bergman (IRF, Institute for Radio Physics, SE), and Doug Liddle (SSTL, Surrey Satellite Technology Ltd, UK).

\section{References}

[1] Burns, J.O. et al., 2012, AdSpR 48, 1942

[2] Haslam, C. G. T., Salter, C. J., Stoffel, H., \& Wilson, W. E., 1982, A\&AS 47, 1

[3] Loeb, A., 2006, SciAm 295, 46

[4] SURO-LC Proposal for ESA S-Class Call, 2012, http://www.astron.nl/surolc/ 